\title{
ATLAS Measurements of Electroweak Boson Production Cross Sections
}

\author{
Christian Goeringer on behalf of the ATLAS collaboration
}

\begin{abstract}
Electroweak production cross-sections are presented measured with a dataset with an integrated luminosity of $36 \mathrm{pb}^{-1}$ to $1 \mathrm{fb}^{-1}$ taken at the ATLAS detector at the LHC with pp-collisions at $\sqrt{s}=7 \mathrm{TeV}$. In particular are shown, inclusive $\mathrm{W}$ and $\mathrm{Z}$ measurements as well as $\mathrm{W}$ and $\mathrm{Z}$ in association with jets and their cross-section ratio. Also discussed are crosssection measurements of the diboson processes WW, WZ, ZZ, Z+gamma and W+gamma. All measurements are compared to Standard Model (SM) theory predictions and no deviation was observed. Limits were derived on anomalous like Triple Gauge Couplings (aTGCs).
\end{abstract}

Keywords: W boson, $\mathrm{Z}$ boson, Diboson, W+jets, Z+jets, proton proton scattering, cross section, PDF, TGC PACS: $12.15 .-\mathrm{y}, 14.70 . \mathrm{Fm}, 14.70 . \mathrm{Hp}$

In pp collisions at $\sqrt{s}=7 \mathrm{TeV}$ using the ATLAS detector, $\mathrm{W}$ and $\mathrm{Z}$ production cross-sections are measured which are sensitive to parton density functions (PDFs) and lepton universality. The measured $\mathrm{W}+\mathrm{jets}$ and $\mathrm{Z}+\mathrm{jets}$ crosssections as well as the cross-section ratio $\mathrm{W}+1$ jet over $\mathrm{Z}+1$ jet provide insights into QCD models implemented into Monte Carlo (MC) generators. Furthermore cross-sections for diboson processes (WW, WZ, ZZ and W/Z+gamma) are measured which are sensitive to possible triple gauge couplings beyond the standard model. All measurements are compared to theory predictions which are available up to next-to-next-to-leading order (NNLO). For the WW, WZ and $\mathrm{ZZ}$ measurements, the 2011 dataset up to June with an integrated luminosity of $1 \mathrm{fb}^{-1}$ was used. The other results are based on the 2010 dataset of about $36 \mathrm{pb}^{-1}$. In all measurements, only decay channels in electrons and muons are considered and the term leptons is used when there is no differentiation necessary.

The ATLAS detector is a multi-purpose detector described in Ref. [1]. Its major sub detectors are a tracking system (ID), an electromagnetic and hadronic calorimeter as well as a muon system (MS). The major requirements used to select events containing $\mathrm{W}$ or $\mathrm{Z}$ bosons decaying into electrons or muons are given below. Electron candidates with $\eta<2.47$ are required to deposit at least $20 \mathrm{GeV}$ of transverse energy in the EM calorimeter. Furthermore a track measured in the ID is required to point towards this energy deposition. So called "forward" electrons with $2.5<|\eta|<4.9$ are measured outside of the ID coverage, therefore they are identified by electromagnetic clusters only. Electrons in the transition region $1.375<|\eta|<1.52$ are not used at present. Muon candidates consist of a combination of one track in the ID and MS combined within $\eta<2.4$. Electron and muon candidates have to have a transverse momentum $p_{T}$ greater than $20 \mathrm{GeV}$ if not stated otherwise. Photons are identified by electromagnetic clusters with no ID track pointing towards them. Jets are defined using the anti- $\mathrm{k}_{T}$-algorithm with $R=0.4$.

For the selection of so-called "central" Z candidates, two oppositely-charged "central" electrons are required. In case of the so-called "forward" selection in the electron channel, one "central" electron as well as one "forward" electron are required. Since there is no tracking information on the forward electron, there is no opposite charge requirement applied. In both cases, a $\mathrm{Z}$ candidate mass in the window $66<M_{Z}<116$ is required. For $\mathrm{W}$ candidates, events are selected which contain at least one lepton as well as missing transverse energy $\left(E_{m i s s}^{T}\right)$ of at least $25 \mathrm{GeV}$. The transverse mass $\left(M_{T}\right)$ of the $\mathrm{W}$ candidate has to be greater than $40 \mathrm{GeV}$. These selection criteria for $\mathrm{W}$ and $\mathrm{Z}$ candidates describe the basic selection used in almost all analysis described here, in the following only deviations are reported.

The background is estimated using different methods. Usually, the background from QCD processes, like di-jets, is estimated via data-driven methods, while the background from electroweak processes is taken from MC.

Fiducial and total cross-sections are measured for the $\mathrm{W}$ and $\mathrm{Z}$ production, defined as $\sigma^{\text {fid }}(\mathrm{W} / \mathrm{Z} \rightarrow l \mathrm{v} / \mathrm{ll})=\frac{N-B}{C_{W / Z} L_{\text {int }}}$ and $\sigma(W / Z \rightarrow l v / l l)=\frac{\sigma^{f i d}(W / Z \rightarrow l v / l l)}{A_{W / Z}}$ respectively. $N$ and $B$ denote the number of candidate and background events. The fiducial volume of the fiducial cross-section is defined by the cuts on the lepton $\eta$ and $p_{T}$ and either the cut on $M_{Z}$ or the cuts on $E_{m i s s}^{T}$ and $M_{T}$ for the $\mathrm{W}$ cross-section. In the total cross-section measurements, this fiducial acceptance is corrected for by $A_{W / Z}$. The efficiency $C_{W / Z}$ corrects for any inefficiencies due to trigger, reconstruction and identification. The uncertainties on the cross-section measurements are dominated by the luminosity uncertainty 

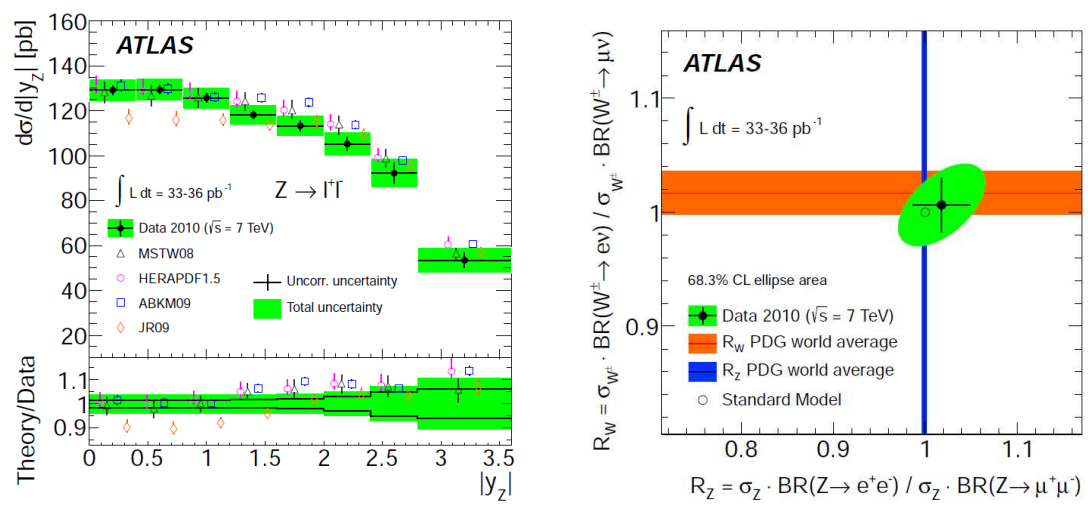

FIGURE 1. Distribution of the cross-section $Z \rightarrow l l$ as a function of $\mathrm{Z}$ rapidity (left panel) [2]. The data is compared to three different NNLO PDF predictions. In the right panel, the ratios of cross-section times branching ratio $W^{ \pm} \rightarrow e v_{e}$ over $W^{ \pm} \rightarrow \mu v_{e}$ as well as the corresponding ratio for the $\mathrm{Z}$ is shown. The data is compared to the PDG world average and the SM expectation of lepton universality.

of $3.4 \%$ and in the case of total cross-section measurements also by the theoretical uncertainty on the acceptance. Experimental uncertainties are $1 \%$ to $2 \%$ except for the forward $\mathrm{Z}$ measurements.

Since the measured W/Z cross-sections [2] in the electron and muon decay channels are consistent, they are combined. Fig. 1 (left panel) shows the $\mathrm{Z}$ cross-section as a function of $\mathrm{Z}$ rapidity. Within uncertainties, data and NNLO theory predictions using MSTW2008 and HERA1.5 PDFs agree while ABKM09 and JR09 seem to model the data less well. To exploit the PDF sensitivity, this measurement is planned to be used as input to upcoming PDF fits. The same is true for the corresponding W measurements as well as various other cross-section and cross-section ratio measurements which are not shown here: decay channel separated and $\mathrm{W}$ charge separated measurements and the ratios of various $\mathrm{W}^{ \pm} / \mathrm{Z}$ cross-sections. Fig. 1 (right panel) shows the ratio of the cross-section times branching ratio $W^{ \pm} \rightarrow e v_{e}$ over $W^{ \pm} \rightarrow \mu v_{e}$ as well as the corresponding ratio for the $\mathrm{Z}$. The ratios are compatible with the SM expectations of lepton universality. The ATLAS measurement on the $\mathrm{W}$ ratio has a total uncertainty of $2.4 \%$ which is competitive with the PDG world average uncertainty of $1.9 \%$.

Also measurements of the $\mathrm{W} / \mathrm{Z}+\mathrm{jets}$ cross-sections have been made $[3,4]$. These measurements are on the one hand an import test of QCD and MC generators and on the other hand an important background to many other ATLAS analysis. The selection of the $\mathrm{W}$ and $\mathrm{Z}$ candidates corresponds to the selection in the inclusive case described before. Additionally, jets with $\eta<2.8$ are and a $p_{T}$ of more than $30 \mathrm{GeV}$ (Z+jets) respectively $20 \mathrm{GeV}$ (W+jets) considered. For both channels, differential cross-sections as a function of the number of jets, the ratio $\frac{N_{j e t s}}{N_{\text {jets }}-1}$, leading and second leading $p_{T}$ were measured. In the $\mathrm{W}+\mathrm{jets}$ case, additionally differential cross-sections were measured as a function of the third and fourth leading jet as well as of $H_{T}$, the scalar sum of the $p_{T}$ of all jets, the lepton and the neutrino $\left(E_{\text {miss }}^{T}\right)$. The data are compared to predictions of different MC generators as well as NLO theory and agrees within uncertainties. A dedicated analysis to measure the cross-section ratio of $\mathrm{W}+1$ jet over $\mathrm{Z}+1$ jet was also carried out. It is the first measurement of its kind and already archived a precision of 5\% in the fiducial cross-section ratio. Also here the data and MC predictions agree within uncertainties.

Measurements of diboson production cross-sections have also been carried out, namely WW [6], WZ [7], ZZ [8], and $\mathrm{W} / \mathrm{Z}+\gamma$ [9]. In all diboson analysis, the $\mathrm{W}$ and $\mathrm{Z}$ electron and muon decay channels are considered. For the combined final state, all possible combinations of electrons and muons are measured, e.g., $W Z \rightarrow e e \mu, e e e, \mu \mu e, \mu \mu \mu$. The selection criteria for $\mathrm{W}$ and $\mathrm{Z}$ candidates follow the ones in the inclusive analysis. To select WW events, two leptons and an $E_{\text {miss }}^{T}$ threshold which varies with the final state configuration from $25 \mathrm{GeV}(e \mu)$ to $45 \mathrm{GeV}(\mu \mu)$ is required. Furthermore, events with additional jets above $30 \mathrm{GeV}$ and $\eta<4.5$ are rejected to suppress $\mathrm{W}+\mathrm{jets}$ and $t \bar{t}$ background. In case of two same-flavour oppositely-charged leptons, they are required to be outside of a mass window of $15 \mathrm{GeV}$ around the $\mathrm{Z}$ pole mass to suppress $\mathrm{Z}$ background.

For the WZ event selection, the $\mathrm{Z}$ candidate is required to have a mass close to the pole mass $\left|M_{Z}-M_{l l}\right|<10 \mathrm{GeV}$, while for the $\mathrm{W}$ candidate the requirements are $E_{\text {miss }}^{T}>25 \mathrm{GeV}$ and $M_{T}>20 \mathrm{GeV}$. To select $\mathrm{ZZ}$ events, two pairs of oppositely-charged leptons of same flavor with $66 \mathrm{GeV}<M_{l l}<116 \mathrm{GeV}$ each are required. This signature suppresses background very successfully, 12 signal events with an background expectation of $0.3_{-0.3}^{+0.9}(\text { stat })_{-0.3}^{+0.4}($ syst $)$ events were observed. 

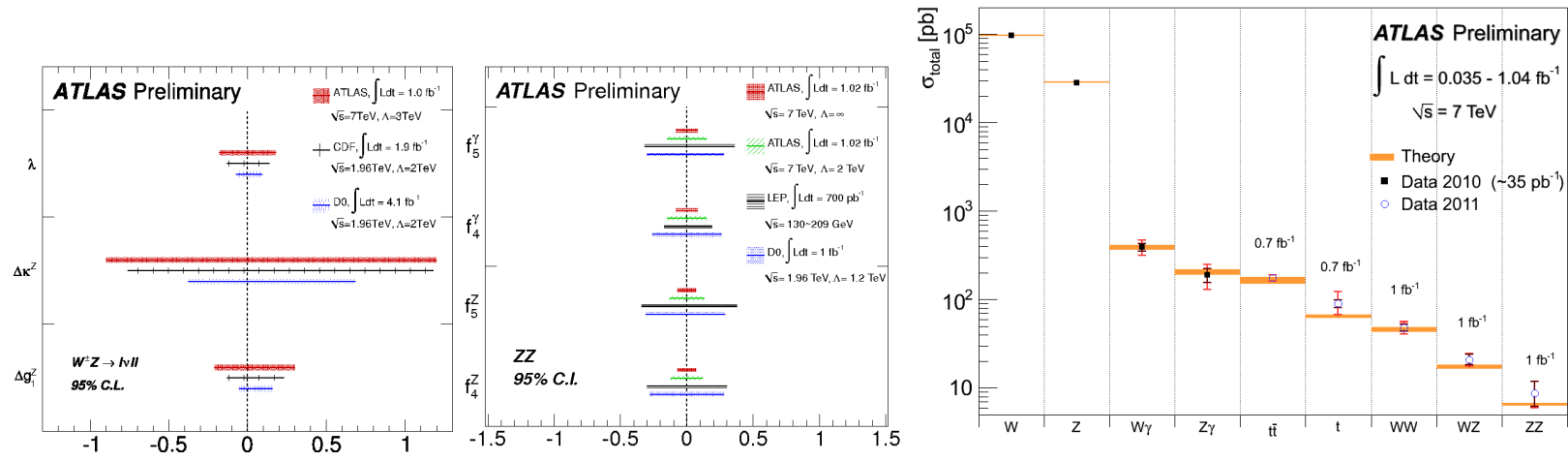

FIGURE 2. The 95\% CL exclusion limits on couplings of the WWZ vertex of ATLAS [7] compared to the CDF and D0 results are shown in the left panel. $\Delta g_{1}^{Z}$ and $\Delta \kappa^{Z}$ represent the deviation from the SM value. In the middle panel, the 95\% CL exclusion limits on couplings of the ZZZ and ZZ $\gamma$ vertices by ATLAS [8], LEP and D0 are shown. An overview on ATLAS measurements of electroweak cross-sections in presented in the right panel.

In case of the $\mathrm{W} / \mathrm{Z}+$ gamma event selection, the isolated photon is required to have an $E_{T}>15 \mathrm{GeV}$ and a minimum distance $\Delta R(l, \gamma)>0.7$ with respect to the lepton(s).

The measured cross-sections of the described diboson processes are compared to NLO theory predictions and agree within uncertainties. The WZ and ZZ measurements are sensitive to triple gauge couplings (TGCs). In the SM, ZZ production proceeds predominantly via the $\mathrm{t}$-channel while the s-channel vertices (ZZZ, ZZ $\gamma$ ) are forbidden. In the WZ case, the WWZ vertex is allowed in the SM. The corresponding gauge couplings are fixed by gauge symmetry. Non-SM gauge couplings can lead to significant enhancements of diboson production. Since the data is consistent with the SM predictions, exclusion limits on anomalous TGCs (aTGCs) are set. To define those aTGC parameters, a more general Lagrangian and a scale factor have been introduced. The details can be found in Ref. $[7,8]$. The coupling constants and their SM values for the WWZ vertex are the following: WWZ: $g_{1}^{Z}=1$, $\kappa^{Z}=1$ and $\lambda=0$. The exclusion limits are shown in Fig. 2 (left panel). For the ZZZ and ZZ $\gamma$ vertices, the values of the couplings, $\left(f_{4}^{b}\right.$ and $f_{5}^{b}$ ) with $b=Z, \gamma$, are zero in the SM. The corresponding exclusion limits are shown in Fig. 2 (middle panel). In both cases, the ATLAS limits (using $1 \mathrm{fb}^{-1}$ of data) are compared to the ones obtained from CDF and D0 (using $1 \mathrm{fb}^{-1}$ to $4.1 \mathrm{fb}^{-1}$ ) and are found to be already competitive.

Fig. 2 (right panel) shows an overview of ATLAS measurements of electroweak cross-sections compared to theory predictions. No deviations from SM expectations are observed.

Copyright CERN for the benefit of the ATLAS Collaboration

\section{REFERENCES}

1. ATLAS Collaboration, The ATLAS Experiment at the CERN Large Hadron Collider, JINST 3 S08003 (2008)

2. ATLAS Collaboration, Measurement of the inclusive $W^{ \pm}$and $\mathrm{Z} / \gamma^{*}$ cross sections in the electron and muon decay channels in pp collisions at $\sqrt{s}=7 \mathrm{TeV}$ with the ATLAS detector, arXiv:1109.5141, submitted to PRD

3. ATLAS Collaboration, Measurement of the production cross section for $W$-bosons in association with jets in pp collisions using $33 \mathrm{pb}^{-1}$ at $\sqrt{\mathrm{s}}=7 \mathrm{TeV}$ with the ATLAS detector, ATLAS-CONF-2011-060, http://cdsweb.cern.ch/record/1344778

4. ATLAS Collaboration, Measurement of the production cross section for $\mathrm{Z} / \gamma^{*}$ in association with jets in pp collisions at $\sqrt{s}=7 \mathrm{TeV}$ with the ATLAS Detector, ATLAS-CONF-2011-042, http://cdsweb.cern.ch/record/1338571

5. ATLAS Collaboration, A measurement of the ratio of the $W+1$ jet to $Z+1$ jet cross sections with ATLAS, arXiv:1108.4908, submitted to PLB

6. ATLAS Collaboration, Measurement of the $W^{+} W^{-}$Production Cross Section in Proton-Proton Collisions at $\sqrt{s}=7 \mathrm{TeV}$ with the ATLAS Detector, ATLAS-CONF-2011-110, http://cdsweb.cern.ch/record/1373412

7. ATLAS Collaboration, Measurement of the $W^{ \pm} Z$ Production Cross Section in Proton-Proton Collisions at $\sqrt{s}=7 \mathrm{TeV}$ with the ATLAS Detector, ATLAS-CONF-2011-099, http://cdsweb.cern.ch/record/1369214

8. ATLAS Collaboration, Measurement of the ZZ Production Cross Section in Proton-Proton Collisions at $\sqrt{s}=7 \mathrm{TeV}$ with the ATLAS Detector, ATLAS-CONF-2011-107, http://cdsweb.cern.ch/record/1372920

9. ATLAS Collaboration, Measurement of $W \gamma$ and $Z \gamma$ production in proton-proton collisions at $\sqrt{s}=7 \mathrm{TeV}$ with the ATLAS Detector, JHEP 09(2011) 072 